\title{
Molecular Spinless Energies of the Modified Rosen-Morse Potential Energy Model
}

\author{
Chun-Sheng Jia, ${ }^{*}$ Xiao-Long Peng, and Su He ${ }^{\dagger}$ \\ State Key Laboratory of Oil and Gas Reservoir Geology and Exploitation, Southwest Petroleum University, \\ Chengdu 610500,People's Republic of China."E-mail: chshjia@263.net \\ ${ }^{\dagger}$ Scientific Research Office, Southwest Petroleum Institute, Chengdu 610500, People's Republic of China \\ Received May 1, 2014, Accepted May 4, 2014
}

\begin{abstract}
We solve the Klein-Gordon equation with the modified Rosen-Morse potential energy model. The bound state energy equation has been obtained by using the supersymmetric shape invariance approach. The relativistic vibrational transition frequencies for the $6^{1} \Pi_{u}$ state of the ${ }^{7} \mathrm{Li}_{2}$ molecule have been computed by using the modified Rosen-Morse potential model. The calculated relativistic vibrational transition frequencies are in good agreement with the experimental RKR values.
\end{abstract}

Key Words : Klein-Gordon equation, Modified Rosen-Morse potential model, Vibrational transition, Lithium dimer

\section{Introduction}

There has been the continuous interest in investigating solutions of the Klein-Gordon equation with some diatomic molecule potential energy models, such as the Morse potential, ${ }^{1,2}$ Rosen-Morse potential, ${ }^{3-5}$ Manning-Rosen potential, ${ }^{6}$ Pöschl-Teller potential, ${ }^{7-9}$ and Deng-Fan potential. ${ }^{10,11}$ As far as we known, the authors in these works did not quantitatively investigated relativistic rotation-vibrational energies for actual diatomic molecules. In recent years, one of present authors and co-workers ${ }^{12-15}$ studied solutions of the Klein-Gordon equation with the improved ManningRosen potential, improved Rosen-Morse potential, improved Tietz potential, and Morse potential, and calculated relativistic vibrational transition frequencies for the $\mathrm{a}^{3} \Sigma_{\mathrm{u}}{ }^{+}$state of the ${ }^{7} \mathrm{Li}_{2}$ molecule, the $3^{3} \Sigma_{\mathrm{g}}{ }^{+}$state of the $\mathrm{Cs}_{2}$ molecule, the $\mathrm{C}^{1} \Pi_{\mathrm{u}}$ state of the $\mathrm{Na}_{2}$ molecule, and the $\mathrm{X}^{1} \Sigma^{+}$state of the ScI molecule.

In 2012, Zhang et al. ${ }^{16}$ introduced the effect of inner-shell radii of two atoms for diatomic molecules into the original Rosen-Morse potential, ${ }^{17}$ and proposed a modified RosenMorse potential energy model,

$$
U_{\mathrm{MRM}}(r)=D_{e}\left(1-\frac{e^{\alpha\left(r_{e}-r_{i j}\right)}+1}{e^{\alpha\left(r-r_{i j}\right)}+1}\right)^{2},
$$

where $r_{i j}=r_{e}-\sqrt{K D_{e} / k_{e}}, D_{e}$ denotes the dissociation energy, $r_{e}$ denotes the equilibrium bond length, and $K_{e}$ presents the equilibrium harmonic vibrational force constant. The parameter $K$ is a dimensionless constant, $K=4.00 .^{18}$ The ability of a potential energy model to reproduce the potential energy curve is judged by agreement with the known potential energy curve determined by the Rydberg-Klein-Rees (RKR) approach. ${ }^{19-21}$ The modified Rosen-Morse potential is superior to the Morse potential and original Rosen-Morse potential in reproducing potential energy curves for six molecule states examined in Ref.. ${ }^{16}$ Recently, with the use of the standard function analysis method, Tang et al. ${ }^{22}$ solved the Schrödinger equation with the modified Rosen-Morse potential model to obtain rotation-vibrational energy spectra for a diatomic molecule, and calculated the vibrational energy levels calculated for the $6^{1} \Pi_{u}$ state of the ${ }^{7} \mathrm{Li}_{2}$ molecule. In terms of the RKR method and the multireference configuration interaction approach, many authors have carried out on investigation on the vibrational levels and the interaction potential curves for the lithium dimer. ${ }^{23-25}$

In this work, we employ the basic concept of the supersymmetric shape invariance approach to study the bound state solutions of the Klein-Gordon equation with the modified Rosen-Morse potential energy model. We also attempt to calculate the relativistic vibrational transition frequencies of the ${ }^{6} \Pi_{u}$ state of the ${ }^{7} \mathrm{Li}_{2}$ molecule and compare the present calculated values with the RKR data.

\section{Bound State Solutions}

The Klein-Gordon equation with a scalar potential $S(r)$ and a vector potential $V(r)$ for the nuclear motion of a diatomic molecule with reduced mass $\mu$ is given by ${ }^{12}$

$$
\left[-\hbar^{2} c^{2} \nabla^{2}+\left(M c^{2}+S(r)\right)^{2}\right] \Psi(r, \theta, \varphi)=[E-V(r)]^{2} \Psi(r, \theta, \varphi),
$$

where $\nabla^{2}$ is the Laplace operator, $E$ denotes the relativistic energy of the quantum system, $c$ is the speed of light, and $\hbar$ $=h / 2 \pi$, $h$ is the Planck constant. We express the wave function as $\Psi(r, \theta, \varphi)=\left(u_{v J}(r) / r\right) Y_{J m}(\theta, \varphi)$, where $Y_{J m}(\theta, \varphi)$ is the spherical harmonic function. Substituting this expression into Eq. (2), we obtain the radial part of the Klein-Gordon equation,

$$
\begin{aligned}
& {\left[-\hbar^{2} c^{2} \frac{d^{2}}{d r^{2}}+2\left(\mu c^{2} S(r)+E_{v J} V(r)\right)+S^{2}(r)-V^{2}(r)+\frac{J(J+1) \hbar^{2} c^{2}}{r^{2}}\right] u_{v J}(r)} \\
& =\left(E_{v J}^{2}-\mu^{2} c^{4}\right) u_{v J}(r)
\end{aligned}
$$


where $v$ and $J$ are the vibrational and rotational quantum numbers, respectively. Taking the equal scalar and vector potentials, $S(r)=V(r)$, Eq. (3) turns to the form

$\left[-\hbar^{2} c^{2} \frac{d^{2}}{d r^{2}}+2\left(\mu c^{2}+E_{v J}\right) V(r)+\frac{J(J+1) \hbar^{2} c^{2}}{r^{2}}\right] u_{v J}(r)=\left(E_{v J}^{2}-\mu^{2} c^{4}\right) u_{v, J}(r)$

Under the nonrelativistic limit, Eq. (4) becomes a Schrödinger equation with the interaction potential $2 V(r)$. In order to make the interaction potential as $V(r)$, not $2 V(r)$ in nonrelativistic limit, we take the scheme proposed by Alhaidari et $a l^{26}$ to rescale the scalar potential $S(r)$ and vector potential $V(r)$, and write Eq. (4) in the form of

$$
\begin{aligned}
& {\left[-\hbar^{2} c^{2} \frac{d^{2}}{d r^{2}}+2\left(\mu c^{2} \frac{S(r)}{2}+E_{v J} \frac{V(r)}{2}+\left(\frac{S(r)}{2}\right)^{2}-\left(\frac{V(r)}{2}\right)^{2}\right)+\frac{J(J+1) \hbar^{2} c^{2}}{r^{2}}\right] u_{v J}(r)} \\
& =\left(E_{v J}^{2}-\mu^{2} c^{2}\right) u_{v J}(r) .
\end{aligned}
$$

Considering the scalar and vector potentials as the modified Rosen-Morse potential, $S(r)=V(r)=U_{\mathrm{MRM}}(r)$, we obtain the following second-order Schrödinger-like equation,

$$
\begin{aligned}
& {\left[-\hbar^{2} c^{2} \frac{d^{2}}{d r^{2}}+\left(\mu c^{2}+E_{v J}\right) D_{e}\left(1-\frac{e^{\alpha r_{e}}+\lambda}{e^{\alpha r}+\lambda}\right)^{2}+\frac{J(J+1) \hbar^{2} c^{2}}{r^{2}}\right] u_{v J}(r)} \\
& =\left(E_{v J}^{2}-\mu^{2} c^{4}\right) u_{v J}(r),
\end{aligned}
$$

where $\lambda=e^{\alpha r_{i j}}$. In the case of $J=0$, we can exactly solve the above equation. When $J \neq 0$, one can only approximately solve it. We apply the Pekeris approximation scheme to deal with the centrifugal term. ${ }^{27}$ The Pekeris approximation approach has been widely used to investigate the analytical solutions of the Klein-Gordon equation with various molecular potential models. ${ }^{11-13}$ We replace the centrifugal potential energy term by the following form ${ }^{22}$

$$
\frac{J(J+1) \hbar^{2} c^{2}}{r^{2}} \approx \gamma\left(c_{0}+c_{1} \frac{1}{e^{\alpha r}+\lambda}+c_{2} \frac{1}{\left(e^{\alpha r}+\lambda\right)^{2}}\right),
$$

where $\gamma=\left(J(J+1) \hbar^{2} c^{2}\right) / r_{e}^{2}$, and the coefficients $c_{0}, c_{1}$, and $c_{2}$ are given $b^{22}$

$$
\begin{aligned}
& c_{0}=1+\frac{1}{\alpha^{2} r_{e}^{2}}\left(3-3 \alpha r_{e}+6 \lambda e^{-\alpha r_{e}}+3 \lambda^{2} e^{-2 \alpha r_{e}}-2 \lambda \alpha r_{e} e^{-\alpha r_{e}}+\lambda^{2} \alpha r_{e} e^{-2 \alpha r_{e}}\right), \\
& c_{1}=\frac{2}{\alpha^{2} r_{e}^{2}}\left(-9 \lambda+3 \lambda \alpha r_{e}-3 e^{\alpha r_{e}}+2 a r_{e} e^{\alpha r_{e}}-9 \lambda^{2} e^{-\alpha r_{e}}-3 \lambda^{3} e^{-2 \alpha r_{e}}-\lambda^{3} a r_{e} e^{-2 \alpha r_{e}}\right),
\end{aligned}
$$

$$
\begin{aligned}
c_{2}=\frac{1}{\alpha^{2} r_{e}^{2}}\left(18 \lambda^{2}+12 \lambda e^{\alpha r_{e}}+3 e^{2 \alpha r_{e}}-2 \lambda \alpha r_{e} e^{\alpha r_{e}}-\alpha r_{e} e^{2 \alpha r_{e}}+12 \lambda^{3} e^{-\alpha r_{e}}\right. \\
\left.+3 \lambda^{4} e^{-2 \alpha r_{e}}+2 \lambda^{3} \alpha r_{e} e^{-\alpha r_{e}}+\lambda^{4} \alpha r_{e} e^{-2 \alpha r_{e}}\right) .
\end{aligned}
$$

Substituting approximation expression (7) into Eq. (6), we rewrite it in the following form

$$
\left[-\frac{d^{2}}{d r^{2}}+\frac{A}{e^{\alpha r}+\lambda}+\frac{B}{\left(e^{\alpha r}+\lambda\right)^{2}} e^{\alpha r}\right] u_{v J}(r)=\varepsilon_{v J} u_{v J}(r) \text {. }
$$

where the parameters $A, B, \varepsilon_{v J}$ are defined as

$$
\begin{aligned}
& A=\frac{1}{\hbar^{2} c^{2} \lambda}\left[\left(\mu c^{2}+E_{v J}\right) D_{e}\left(e^{2 \alpha r_{e}}-\lambda^{2}\right)+\gamma\left(\lambda c_{1}+c_{2}\right)\right], \\
& B=-\frac{1}{\hbar^{2} c^{2} \lambda}\left[\left(\mu c^{2}+E_{v J}\right) D_{e}\left(e^{\alpha r_{e}}+\lambda\right)^{2}+\gamma c_{2}\right], \\
& \varepsilon_{v J}=-\frac{1}{\hbar^{2} c^{2}}\left[\left(E_{v J}^{2}-\mu^{2} c^{4}\right)-\left(\mu c^{2}+E_{v J}\right) D_{e}-\gamma c_{0}\right] .
\end{aligned}
$$

With the use of the supersymmetric shape invariance approach, ${ }^{28-30}$ we solve Eq. (11). The ground-state wave function $u_{0, J}(r)$ is written as

$$
u_{0, J}(r)=\exp \left(-\int W(r) d r\right),
$$

where $W(r)$ is called a superpotential in supersymmetric quantum mechanics. ${ }^{28}$ Substituting expression (15) into Eq. (11) yields the following relation satisfied by the superpotential $W(r)$,

$$
W^{2}(r)-\frac{d W(r)}{d r}=\frac{A}{e^{\alpha r}+\lambda}+\frac{A}{\left(e^{\alpha r}+\lambda\right)^{2}} e^{\alpha r}-\varepsilon_{0, J},
$$

where $\varepsilon_{0, J}$ presents the ground-state energy. We take the superpotential $W(r)$ in the form

$$
W(r)=C_{1}+\frac{C_{2}}{e^{\alpha r}+\lambda},
$$

where $C_{1}$ and $C_{2}$ are two constants. Substituting the above expression into expression (15), we obtain the following expression for the ground-state wave function $u_{0, J}(r)$,

$$
u_{0, J}(r)=e^{-C_{1} r}\left(1+\lambda e^{-\alpha r}\right)^{\frac{C_{2}}{\alpha \lambda}} .
$$

For the bound state solutions, the wave function $u_{v J}(r)$ should satisfy the boundary conditions: $u_{v J}(\infty)=0$ and $u_{v J}(0)$ is limitary. These regularity conditions leads us to have $C_{1}<0$ and $C_{2}<0$.

In terms of expression (17), we can construct a pair of supersymmetric partner potentials $U_{-}(r)$ and $U_{+}(r)$,

$$
\begin{aligned}
& U_{-}(r)=W^{2}(r)-\frac{d W(r)}{d r^{2}}=C_{1}^{2}+\frac{2 C_{1} C_{2}+\frac{C_{2}^{2}}{\lambda}}{e^{\alpha r}+\lambda}+\frac{\alpha C_{2}-\frac{C_{2}^{2}}{\lambda}}{\left(e^{\alpha r}+\lambda\right)^{2}} e^{\alpha r}, \\
& U_{+}(r)=W^{2}(r)+\frac{d W(r)}{d r^{2}}=C_{1}^{2}+\frac{2 C_{1} C_{2}+\frac{C_{2}^{2}}{\lambda}}{e^{\alpha r}+\lambda}+\frac{-\alpha C_{2}-\frac{C_{2}^{2}}{\lambda}}{\left(e^{\alpha r}+\lambda\right)^{2}} e^{\alpha r},
\end{aligned}
$$

Making a comparison of Eq. (19) with Eq. (16), we have the following three relationships

$$
\begin{gathered}
C_{1}^{2}=-\varepsilon_{0, J} \\
2 C_{1} C_{2}+\frac{C_{2}^{2}}{\lambda}=A, \\
\alpha C_{2}-\frac{C_{2}^{2}}{\lambda}=B .
\end{gathered}
$$


Solving Eqs. (22) and (23), we obtain

$$
\begin{gathered}
C_{1}=\frac{A}{2 C_{2}}-\frac{C_{2}}{2 \lambda}, \\
C_{2}=\frac{\alpha \lambda}{2}\left(1-\sqrt{1-\frac{4 B}{\alpha^{2} \lambda}}\right)
\end{gathered}
$$

Substituting expression $C_{1}=\frac{A}{2 C_{2}}-\frac{C_{2}}{2 \lambda}$ into expressions (19) and (20) and using Eq. (22), we can rewrite the two supersymmetric partner potentials $U_{-}(r)$ and $U_{+}(r)$ as follows

$$
\begin{gathered}
U_{-}(r)=\left(\frac{A}{2 C_{2}}-\frac{C_{2}}{2 \lambda}\right)^{2}+\frac{A}{e^{\alpha r}+\lambda}+\frac{\alpha C_{2}-\frac{C_{2}^{2}}{\lambda}}{\left(e^{\alpha r}+\lambda\right)^{2}} e^{\alpha r}, \\
U_{+}(r)=\left(\frac{A}{2 C_{2}}-\frac{C_{2}}{2 \lambda}\right)^{2}+\frac{A}{e^{\alpha r}+\lambda}+\frac{-\alpha C_{2}-\frac{C_{2}^{2}}{\lambda}}{\left(e^{\alpha r}+\lambda\right)^{2}} e^{\alpha r} .
\end{gathered}
$$

With the use of expressions (26) and (27), we can yield the following relationship

$$
U_{+}\left(r, a_{0}\right)=U_{-}\left(r, a_{1}\right)+R\left(a_{1}\right)
$$

where $a_{0}=C_{2}, a_{1}$ is a function of $a_{0}$, i.e., $a_{1}=h\left(a_{0}\right)=a_{0}+\alpha \lambda$, and the reminder $R\left(a_{1}\right)$ is independent of $r, R\left(a_{1}\right)=\left(\frac{A}{2 a_{0}}-\frac{a_{0}}{2 \lambda}\right)^{2}-$ $\left(\frac{A}{2 a_{1}}-\frac{a_{1}}{2 \lambda}\right)^{2}$. Eq. (28) implies that the supersymmetric partner potentials $U_{-}(r)$ and $U_{+}(r)$ possess the shape invariance. Using the shape invariance approach, ${ }^{29}$ one can exactly determine their energy spectra. The energy spectra of the potential $U_{-}(r)$ are given by

$$
\varepsilon_{o, J}^{(-)}=0,
$$

$$
\begin{aligned}
\varepsilon_{v J}^{(-)} & =\sum_{k=1}^{v} R\left(a_{k}\right)=R\left(a_{1}\right)+R\left(a_{2}\right)+\cdots+R\left(a_{v}\right) \\
& =\left(\frac{A}{2 a_{0}}-\frac{a_{0}}{2 \lambda}\right)^{2}-\left(\frac{A}{2 a_{1}}-\frac{a_{1}}{2 \lambda}\right)^{2}+\left(\frac{A}{2 a_{1}}-\frac{a_{1}}{2 \lambda}\right)^{2}-\left(\frac{A}{2 a_{2}}-\frac{a_{2}}{2 \lambda}\right)^{2} \\
& +\cdots+\left(\frac{A}{2 a_{v-1}}-\frac{a_{v-1}}{2 \lambda}\right)^{2}-\left(\frac{A}{2 a_{v}}-\frac{a_{v}}{2 \lambda}\right)^{2} \\
& =\left(\frac{A}{2 a_{0}}-\frac{a_{0}}{2 \lambda}\right)^{2}-\left(\frac{A}{2 a_{v}}-\frac{a_{v}}{2 \lambda}\right)^{2} \\
& =\left(\frac{A}{2 C_{2}}-\frac{C_{2}}{2 \lambda}\right)^{2}-\left(\frac{A}{2\left(C_{2}+w \alpha \lambda\right)}-\frac{C_{2}+v \alpha \lambda}{2 \lambda}\right)^{2}
\end{aligned}
$$

where the quantum number $v=0,1,2, \cdots$. From Eqs. (11), (16) and (26), we obtain the following relationship for $\varepsilon_{v J}$,

$$
\varepsilon_{v J}=\varepsilon_{v J}^{(-)}+\varepsilon_{0, J} .
$$

Substituting expressions (30) and (21) into Eqs. (31) and considering $C_{1}=A / 2 \mathrm{C}_{2}-C_{2} / 2 \lambda$, we obtain the following expression for $\varepsilon_{v J}$,

$$
\varepsilon_{v J}=\left(\frac{A}{2\left(C_{2}+v \alpha \lambda\right)}-\frac{C_{2}+v \alpha \lambda}{2 \lambda}\right)^{2} .
$$

Substituting expression (25) into expression (32), we have

$$
\varepsilon_{v J}=-\alpha^{2}\left(\frac{\frac{A}{\alpha^{2} \lambda}}{2 v+1-\sqrt{1-\frac{4 B}{\alpha^{2} \lambda}}}-\frac{2 v+1-\sqrt{1-\frac{4 B}{\alpha^{2} \lambda}}}{4}\right)^{2} .
$$

Substituting expressions (12) and (13) into expression (33) and using $\varepsilon_{v J}=-\frac{1}{\hbar^{2} c^{2}}\left(E_{v J}^{2}-\mu^{2} c^{4}\right)-\left(\mu c^{2}+E_{v J}\right) D_{e}-\gamma c_{0}$, we obtain the relativistic rotation-vibrational energy eigenvalue equation for the diatomic molecule in the presence of equal scalar and vector modified Rosen-Morse potential energy models,

$$
\begin{aligned}
& E_{v J}^{2}-\mu^{2} c^{4}=\left(\mu c^{2}+E_{v J}\right) D_{e}+\frac{J(J+1) \hbar^{2} c^{2}}{r_{e}^{2}} c_{0} \\
& -\alpha^{2} \hbar^{2} c^{2}\left[\frac{\frac{J(J+1)}{\alpha^{2} r_{e}^{2} \lambda^{2}}\left(\lambda c_{1}+c_{2}\right)+\frac{\left(\mu c^{2}+E_{v J}\right) D_{e}\left(e^{2 \alpha r_{e}}-\lambda^{2}\right)}{\hbar^{2} c^{2} \alpha^{2} \lambda^{2}}}{2 v+1-\sqrt{1+\frac{4 J(J+1)}{\alpha^{2} r_{e}^{2} \lambda^{2}} c_{2}+\frac{4\left(\mu c^{2}+E_{v J}\right) D_{e}\left(e^{\alpha r_{e}}+\lambda\right)^{2}}{\hbar^{2} c^{2} \alpha^{2} \lambda^{2}}}}\right. \\
& \left.-\frac{2 v+1-\sqrt{1+\frac{4 J(J+1)}{\alpha^{2} r_{e}^{2} \lambda^{2}} c_{2}+\frac{4\left(\mu c^{2}+E_{v J}\right) D_{e}\left(e^{\alpha r_{e}}+\lambda\right)^{2}}{\hbar^{2} c^{2} \alpha^{2} \lambda^{2}}}}{4}\right]^{2}
\end{aligned}
$$

where $v=0,1,2,3, \ldots, \lambda=e^{\alpha r_{i j}}$, and we have made a replacement for $\gamma=\frac{J(J+1) \hbar^{2} c^{2}}{r_{e}^{2}}$.

When $J=0$, we obtain the relativistic vibrational energy eigenvalue equation for the diatomic molecule with equal scalar and vector modified Rosen-Morse potentials,

$$
\begin{aligned}
& E_{v, J}^{2}-\mu^{2} c^{4}=\left(\mu c^{2}+E_{v J}\right) D_{e} \\
&-\alpha^{2} \hbar^{2} c^{2}\left[\frac{\frac{\left(\mu c^{2}+E_{v J}\right) D_{e}\left(e^{2 \alpha r_{e}}-\lambda^{2}\right)}{\hbar^{2} c^{2} \alpha^{2} \lambda^{2}}}{2 v+1-\sqrt{1+\frac{4\left(\mu c^{2}+E_{v J}\right) D_{e}\left(e^{\alpha r_{e}}+\lambda\right)^{2}}{\hbar^{2} c^{2} \alpha^{2} \lambda^{2}}}}\right. \\
&\left.-\frac{2 v+1-\sqrt{1+\frac{4\left(\mu c^{2}+E_{v J}\right) D_{e}\left(e^{\alpha r_{e}}+\lambda\right)^{2}}{\hbar^{2} c^{2} \alpha^{2} \lambda^{2}}}}{4}\right]
\end{aligned}
$$

With the use of the superpotential given in expression (17) and the ground-state wave function given in expression (18), 
one can determine the excited state wave functions by employing the explicit recursion operator approach. ${ }^{31,32}$

\section{Discussion}

The force constant $k_{e}$ is defined as the second derivative of the potential energy function $U(r)$ for a diatomic molecule, namely $k_{e}=\left.\frac{d^{2} U(r)}{d r^{2}}\right|_{r=r_{e}}$. Using this definition and the relation $k_{e}=4 \pi^{2} \mu c^{2} \omega_{e}^{2}$, we deduce the following expression for the potential parameter $\alpha$ appearing in the modified RosenMorse potential,

$$
\alpha=\pi c \omega_{e} \sqrt{\frac{2 \mu}{D_{e}}}+\frac{1}{r_{e}-r_{i j}} W\left(\pi c \omega_{e} \sqrt{\frac{2 \mu}{D_{e}}}\left(r_{e}-r_{i j}\right) e^{-\pi c \omega_{e} \sqrt{\frac{2 \mu}{D_{e}}}\left(r_{e}-r_{i j}\right)}\right),
$$

where $\omega_{e}$ denotes the equilibrium harmonic vibrational reciprocal wavelength, and $W$ is the Lambert $W$ function, which satisfies $z=W(z) e^{W(z)}$.

We consider the ${ }^{6} \Pi_{u}$ state of the ${ }^{7} \mathrm{Li}_{2}$ molecule. The experimental values of $D_{e}, r_{e}$, and $\omega_{e}$ for the ${ }^{7} \mathrm{Li}_{2} 6^{1} \Pi_{\mathrm{u}}$ state are taken from the literature ${ }^{23} \mathrm{~cm}^{-1}, \AA$, and $\mathrm{cm}^{-1}$. Taking these experimental data as inputs and applying expression (36), we can determine the value of the parameter $\alpha$ in the modified Rosen-Morse potential. The experimental RKR data points for the $6^{1} \Pi_{u}$ state of ${ }^{7} \mathrm{Li}_{2}$ are shown in Figure 1, which also contains the curves reproduced by employing the four three-parameter potential models: modified RosenMorse potential, Rosen-Morse potential, ${ }^{17,33}$ Morse potential, ${ }^{34}$ and Frost-Musulin potential. ${ }^{33,35}$ The Rosen-Morse, Morse, and Frost-Musulin potential functions are given by, respectively,

$$
\begin{aligned}
& U_{\mathrm{RM}}(r)=D_{e}\left(1-\frac{e^{\alpha r_{e}}+1}{e^{\alpha r}+1}\right)^{2}, \\
& U_{\mathrm{M}}(r)=D_{e}\left(1-e^{-\beta\left(r-r_{e}\right)}\right)^{2}, \\
& U_{\mathrm{FM}}(r)=D_{e}\left[1-\frac{\left(r+\delta r_{e} r-\delta r_{e}^{2}\right) e^{-\delta\left(r-r_{e}\right)}}{r}\right] .
\end{aligned}
$$

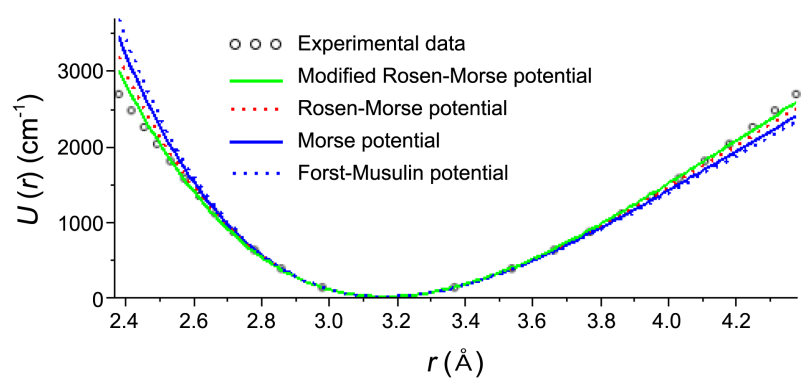

Figure 1. (Color online) RKR data points and four empirical potential energy models for the $6^{1} \Pi_{u}$ state of the ${ }^{7} \mathrm{Li}_{2}$ molecule.
Table 1. A comparison of the calculated relativistic vibrational transition frequencies and experimental RKR values for the $6^{1} \Pi_{u}$ state of the ${ }^{7} \mathrm{Li}_{2}$ molecule (in units of $\mathrm{cm}^{-1}$ )

\begin{tabular}{ccc}
\hline$v(\rightarrow v-1)$ & RKR value & Present calculation \\
\hline 1 & 249.946 & 250.271 \\
2 & 246.378 & 246.943 \\
3 & 242.873 & 243.592 \\
4 & 239.428 & 240.218 \\
5 & 236.045 & 236.821 \\
6 & 232.723 & 233.400 \\
7 & 229.462 & 229.955 \\
8 & 226.262 & 226.486 \\
9 & 223.124 & 222.992 \\
10 & 220.047 & 219.473 \\
11 & 217.030 & 215.928 \\
\hline
\end{tabular}

An outlook for the range covered by the experimental RKR data points tells us that the modified Rosen-Morse potential is superior to the Rosen-Morse, Morse, and Frost-Musulin potential functions for the $6^{1} \Pi_{u}$ state of the ${ }^{7} \mathrm{Li}_{2}$ molecule.

An available potential function should well model the experimental RKR potential curve, and satisfy the Lippincott criterion, i.e., an average absolute deviation of less than $1 \%$ of the dissociation energy $D_{e}{ }^{36}$ The average absolute deviation is defined as $\sigma_{\mathrm{av}}=100 \Sigma\left(\left|U_{\exp }(r)-U_{\text {calc }}(r)\right|\right) / N_{p} D_{e}$, where is the number of experimental data points, $U_{\exp }(r)$ and $U_{\text {calc }}(r)$ are the experimentally determined potential and the empirical potential, respectively. The average absolute deviations of the modified Rosen-Morse potential, RosenMorse potential, Morse potential, and Frost-Musulin potential for the $6^{1} \Pi_{u}$ state of the ${ }^{7} \mathrm{Li}_{2}$ molecule from the RKR potential reported by Grochola et al. ${ }^{23}$ are $0.648 \%$ of $D_{e}$, $1.053 \%$ of $D_{e}, 1.901 \%$ of $D_{e}$, and $2.64 \%$ of $D_{e}$, respectively. These deviation values show that the modified Rosen-Morse potential is best for the examined four potential models in reproducing the potential energy curve for the $6^{1} \Pi_{u}$ state of the ${ }^{7} \mathrm{Li}_{2}$ molecule.

By employing energy eigenvalue Eq. (35), we calculate relativistic vibrational transition frequencies for the $6^{1} \Pi_{u}$ state of the ${ }^{7} \mathrm{Li}_{2}$ molecule. The present calculated values are given in Table 1 , in which we also present the RKR values taken from the literature. ${ }^{23}$ From Table 1, we observe that the relativistic vibrational transition frequencies obtained by using the modified Rosen-Morse potential model are in good agreement with the RKR data.

\section{Conclusions}

In this work, we have investigated the bound state solutions of the Klein-Gordon equation with the Morse potential energy model. The energy eigenvalue equation has been obtained using the supersymmetric shape invariance method. We calculate the relativistic vibrational transition frequencies for the $6^{1} \Pi_{u}$ state of the ${ }^{7} \mathrm{Li}_{2}$ molecule. The relativistic vibrational transition frequencies predicted with the modified Rosen-Morse potential model are in good agreement with the experimental RKR data. 
Acknowledgments. Publication cost of this paper was supported by the Korean Chemical Society.

\section{References}

1. Sun, H. Bull. Korean Chem. Soc. 2011, 32, 4233.

2. Bayrak, O.; Soylu, A.; Boztosun, I. J. Math. Phys. 2010, 51, 112301.

3. Yi, L. Z.; Diao, Y. F.; Liu, J. Y.; Jia, C. S. Phys. Lett. A 2004, 333, 212.

4. Ibrahim, T. T.; Oyewumi, K. J.; Wyngaardt, S. M. Eur. Phys. J. Plus 2012, 127, 100.

5. Qiang, W. C.; Sun, G. H.; Dong, S. H. Ann. Phys. (Berlin) 2012, 524,360 .

6. Wei, G. F.; Zhen, Z. Z.; Dong, S. H. Cent. Eur. J. Phys. 2009, 7, 175.

7. Qiang, W. C.; Dong, S. H. Phys. Lett. A 2008, 372, 4789.

8. Koçak, G.; Taşkın, F. Ann. Phys. (Berlin) 2010, 522, 802.

9. Xu, Y.; He, S.; Jia, C. S. Phys. Scr. 2010, 81, 045001.

10. Dong, S. H. Commun. Theor. Phys. 2011, 55, 969.

11. Oluwadare, O. J.; Oyewumi, K. J.; Akoshile, C. O.; Babalola, O. A. Phys. Scr. 2012, 86, 035002.

12. Jia, C. S.; Cao, S. Y. Bull. Korean Chem. Soc. 2013, 34, 3425.

13. Liu, J. Y.; Du, J. F.; Jia, C. S. Eur. Phys. J. Plus 2013, 128, 139.

14. Chen, T.; Lin, S. R.; Jia, C. S. Eur. Phys. J. Plus 2013, 128, 69.

15. Jia, C. S.; Chen, T.; He, S. Phys. Lett. A 2013, 377, 682.

16. Zhang, G. D.; Liu, J. Y.; Zhang, L. H.; Zhou, W.; Jia, C. S. Phys. Rev. A 2012, 86, 062510 .
17. Rosen, N.; Morse, P. M. Phys. Rev. 1932, 42, 210.

18. Frost, A. A.; Musulin, B. J. Am. Chem. Soc. 1954, 76, 2045.

19. Rydberg, R. Z. Phys. 1933, 80, 514.

20. Klein, O. Z. Phys. 1932, 76, 226.

21. Rees, A. L. G. Proc. Phys. Soc. 1947, 59, 998.

22. Tang, H. M.; Liang, G. C.; Zhang, L. H.; Zhao, F.; Jia, C. S. Can. J. Chem. 2014, $92,341$.

23. Grochola, A.; Jastrzebski, W.; Kowalczyk, P. Mol. Phys. 2008, $106,1375$.

24. Jędrzejewski-Szmek, Z.; Grochola, A.; Jastrzebski, W.; Kowalczyk, P. Chem. Phys. Lett. 2007, 444, 229.

25. Li, D.; Xie, F.; Li, L.; Lazoudis, A.; Lyyra, A. M. J. Mol. Spectrosc. 2007, 246, 180.

26. Alhaidari, A. D.; Bahlouli, H.; Al-Hasan, A. Phys. Lett. A 2006, $349,87$.

27. Pekeris, C. L. Phys. Rev. 1934, 45, 98.

28. Witten, E. Nucl. Phys. B 1981, 185, 513.

29. Gendenshtein, L. E. Sov. Phys.-JETP Lett. 1983, 38, 356.

30. Cooper, F.; Khare, A.; Sukhatme, U. Phys. Rep. 1995, 251, 267.

31. Dabrowska, J. W.; Khare, A.; Sukhatme, U. P. J. Phys. A: Math. Gen. 1988, 21, L195.

32. Jia, C. S.; Wang, X. G.; Yao, X. K.; Chen, P. C.; Xiao, W. J. Phys. A: Math. Gen. 1998, 31, 4763.

33. Morse, M. Phys. Rev. 1929, 34, 57.

34. Frost, A. A.; Musulin, B. J. Chem. Phys. 1954, 22, 1017.

35. Jia, C. S.; Diao, Y. F.; Liu, X. J.; Wang, P. Q.; Liu, J. Y.; Zhang, G. D. J. Chem. Phys. 2012, 137, 014101.

36. Steele, D.; Lippincott, E. R.; Vanderslice, J. T. Rev. Mod. Phys. 1962, 34, 239 